\title{
Synthesis of Substituted N-Alkylamines in Aqueous Media
}

\author{
Avtandil Talybov ${ }^{1}$, Eldar Mamedbeyli ${ }^{1}$, Vaqif Abbasov ${ }^{1}$, Yusif Abdullayev ${ }^{2}$, Konstantin Kochetkov $^{3}$ \\ ${ }^{1}$ Mamedaliyev Institute of Petrochemical Processes, Azerbaijan National Academy of Sciences, Baku, Azerbaijan \\ ${ }^{2}$ Qafqaz University, Baku, Azerbaijan \\ ${ }^{3}$ Nesmeyanov Institute of Organoelement Compounds, Russian Academy of Sciences, Moscow, Russia \\ Email: atalybov@rambler.ru, yabdullayev@qu.edu.az
}

Received June 12, 2012; revised August 1, 2012; accepted August 18, 2012

\begin{abstract}
Synthesis of $\mathrm{N}$-substituted aminopentanes based on the reaction of the amines with 1-brompentanes ( $n$-and iso-structure) and amino (bis-amino) hydroxy compounds via opening reaction of epoxides with amines environmentally friendly practices in water medium has been developed. Structure of obtained compounds by elemental analysis and IR-, ${ }^{1} \mathrm{H}$ and ${ }^{13} \mathrm{C}$ NMR-spectroscopy were confirmed.
\end{abstract}

Keywords: N-Alkylamines; Ionic Liquids; Amino Alcohols; Antibacterial Properties

\section{Introduction}

Modern direction of chemistry and chemical technology includes the development of new, environmentally safe chemical processes. Currently, the synthesis of nitro compounds containing different functional groups environmentally friendly methods in "green" solvents such as ionic liquids or water has attracted special attention of researchers [1-5]. Water use has several advantages such as simplicity of implementation, low cost, high efficiency in many organic reactions involving water soluble substrates, fire safety. Improvement general methods of synthesis based on the available raw materials in aqueous medium are very relevant [6-8]. Substituted diamines were used for the synthesis of azacrown ethers [9], and polymers for special purposes [10], stable carbines [11], silylenes [12] and other stable ions. They are used as ligands in asymmetric synthesis of valuable synthetic and natural products [13] and also for the preparation of complexes with metals such as magnesium, copper, silver, palladium and platinum. The antitum or properties of physiologically active complexes of platinum (II) with diamines have been studied in detail and are widely used in medical practice [14].

\section{Experimental}

IR spectra of the compounds were recorded on a UR-20 spectrometer in the $4000-400 \mathrm{~cm}^{-1} .{ }^{1} \mathrm{H}$ and ${ }^{13} \mathrm{C}$ NMR recorded on a Bruker-300 (300 MHz), solvent $\mathrm{CDCI}_{3}$ and $\mathrm{D}_{2} \mathrm{O}$, chemical shifts are given relative to TMS. Massspectra were obtained on a mass spectrometer VG$7070 \mathrm{E}$ (ionizing voltage $70 \mathrm{eV}$ ). Chromatographic analysis of reaction mixtures and determination of purity of the synthesized compounds was performed on a chromatograph LXM MD-8, a glass column $(2000 \times 3 \mathrm{~mm})$ (10\%-Apiezon on Chromosorb G), carrier gas-helium (40 $\mathrm{cm}^{3} / \mathrm{min}$ ), katarometer, column temperature $-150^{\circ} \mathrm{C}$, the evaporator $-200^{\circ} \mathrm{C}$.

N-Pentylamines( $\left.\mathrm{III}_{\mathrm{a}-\mathrm{j}}\right)$, Common methods of synthesis.

To a solution of $5 \mathrm{mmol}$ of amines $\left(\mathrm{I}_{\mathrm{a}-\mathrm{e}}\right)$ in $5-7 \mathrm{ml}$ of water was added $1 \mathrm{mmol} 1$-brom-pentane $\left(\mathrm{II}_{\mathrm{a}, \mathrm{b}}\right)$ and stirred at a given temperature $\left(50^{\circ} \mathrm{C}-90^{\circ} \mathrm{C}\right)$ for $6-9 \mathrm{~h}$. The mixture was saturated by $10 \mathrm{~g}$ dry powder $\mathrm{NaOH}$. The organic layer was separated, the aqueous layer extracted with ether. The organic layers were combined, dried over $\mathrm{Na}_{2} \mathrm{CO}_{3}$. After distillation of the solvent, the residue was distilled in a vacuum.

$\beta$-Aminoalcohols, Bis-aminopropane $\left(\mathrm{VI}_{\mathrm{a}-\mathrm{c}}, \mathrm{VII}_{\mathrm{a}-\mathrm{c}}\right)$, General method of synthesis.

To a solution of $1.1-5 \mathrm{mmol}$ amines $\left(\mathrm{I}_{\mathrm{a}-\mathrm{c}}\right)$ in $5-7 \mathrm{ml}$ of water was added $1 \mathrm{mmol}$ of epoxide (II or III) and stirred at a given temperature $\left(50^{\circ} \mathrm{C}-90^{\circ} \mathrm{C}\right)$ for $9 \mathrm{~h}$. The mixture was saturated with $10 \mathrm{~g}$ of dry $\mathrm{NaOH}$. The organic layer was separated, the aqueous layer extracted with ether. The organic layers combine, and dried. After distillation of the solvent, the residue was distilled in a vacuum.

N-Penthyldiethylamin(III $\left.)_{a}\right)$ IR $\left(v, \mathrm{~cm}^{-1}\right): 2910\left(\mathrm{CH}_{3}\right)$, $2840\left(\mathrm{CH}_{2}\right), 1230(\mathrm{CN}) .{ }^{1} \mathrm{H}$ NMR $\left(300 \mathrm{MHz}, \mathrm{D}_{2} \mathrm{O}\right) \delta: 0.9$ - 1.2 d.d $\left(9 \mathrm{H}, \mathrm{CH}_{3}\right), 1.4-1.6 \mathrm{~m}\left(6 \mathrm{H}, \mathrm{CH}_{2}\right), 2.45 \mathrm{~m}(2 \mathrm{H}$, $\left.\mathrm{NCH}_{2}\right), 2.6 \mathrm{q}\left(4 \mathrm{H}, \mathrm{CH}_{2} \mathrm{~N}\right) \cdot{ }^{13} \mathrm{C} \mathrm{NMR}\left(300 \mathrm{MHz}, \mathrm{D}_{2} \mathrm{O}\right) \delta$ : $12\left(\mathrm{CH}_{3}\right), 14\left(\mathrm{CH}_{3}\right), 22\left(\mathrm{CH}_{2}\right), 27\left(\mathrm{CH}_{2}\right), 39\left(\mathrm{CH}_{2}\right), 48$ $\left(\mathrm{CH}_{2} \mathrm{~N}\right), 56\left(\mathrm{NCH}_{2}\right)$. Found, \%: C 75.82, H 14.51; N 9.50. $\mathrm{C}_{9} \mathrm{H}_{21}$-N. Calculated, \%: C 75.54, H 14.67; N 9.78.

N-Pentylpiperidine $\left(\mathrm{III}_{\mathrm{b}}\right)$. Obtained from $17 \mathrm{~g}(0.2 \mathrm{~mol})$ 
piperidine $(1 \mathrm{~b})$ and $6.0 \mathrm{~g}$ (0.04 mol) 1-brompentane (IIa). Yield $4.79(78 \%), b p .65^{\circ} \mathrm{C}$ in $(2 \mathrm{~mm} \mathrm{Hg}), \mathrm{n}_{D}^{20} 1.4221$, $\mathrm{d}_{4}^{20}$ 0.8419. IR( $\left(v, \mathrm{~cm}^{-1}\right): 2900\left(\mathrm{CH}_{3}\right), 2830\left(\mathrm{CH}_{2}\right), 1230$ (CN). ${ }^{1} \mathrm{H}$ NMR $\left(300 \mathrm{MHz}, \mathrm{D}_{2} \mathrm{O}\right) \delta: 1.04 \mathrm{mp}\left(\mathrm{CH}, \mathrm{CH}_{3}\right)$, 1.3 - $1.7\left(12 \mathrm{H}, \mathrm{CH}_{2}\right), 2.3 \mathrm{~m}\left(2 \mathrm{H}, \mathrm{NCH}_{2}\right), 2.4 \mathrm{~s}\left(4 \mathrm{H}, \mathrm{CH}_{2}\right.$ Pip.). ${ }^{13} \mathrm{C}$ NMR (300 MHz, $\left.\mathrm{D}_{2} \mathrm{O}\right) \delta: 14.4\left(\mathrm{CH}_{3}\right), 22.8$ $\left(\mathrm{CH}_{2}\right), 24.9\left(\mathrm{CH}_{3}\right), 26.0\left(\mathrm{CH}_{2}\right), 26.9\left(\mathrm{CH}_{2}\right.$ pip. $), 30.0$ $\left(\mathrm{CH}_{2}\right.$ pip.), $54.8\left(\mathrm{CH}_{2} \mathrm{~N}\right.$ pip.), $59.3\left(\mathrm{NCH}_{2}\right.$ aliph.). Found, \%: C 77.82, H 13.62; N 9.13. $\mathrm{C}_{10} \mathrm{H}_{21} \mathrm{~N}$. Calculated, \%: C 77.42, H 13.55; N 9.03.Yield $11 \mathrm{~g}(77 \%)$, bp. $41^{\circ} \mathrm{C}$ $42^{\circ} \mathrm{C}(20 \mathrm{~mm} \cdot \mathrm{Hg}), \mathrm{n}_{D}^{20} 1.4109, \mathrm{~d}_{4}^{20} 0.7779$.

N-Pentylmorpholine (III $)$, 0.8957. IR $\left(v, \mathrm{~cm}^{-1}\right): 2910$ $\left(\mathrm{CH}_{3}\right), 2850\left(\mathrm{CH}_{2}\right), 1230(\mathrm{C}-\mathrm{N}) .{ }^{1} \mathrm{H}$ NMR $(300 \mathrm{MHz}$, $\left.\mathrm{D}_{2} \mathrm{O}\right) \delta: 1.05 \mathrm{~m}\left(3 \mathrm{H}, \mathrm{CH}_{3}\right), 1.45-1.65 \mathrm{~m}\left(6 \mathrm{H}, \mathrm{CH}_{2}\right), 2.4$ $\mathrm{m}\left(2 \mathrm{H}, \mathrm{NCH}_{2}\right), 2.5 \mathrm{~m}\left(4 \mathrm{H}, \mathrm{CH}_{2} \mathrm{~N}\right), 3.65-3.75 \mathrm{~m}(4 \mathrm{H}$, $\left.\mathrm{OCH}_{2}\right) \cdot{ }^{13} \mathrm{C}$ NMR $\left(300 \mathrm{MHz}, \mathrm{D}_{2} \mathrm{O}\right) \delta: 14\left(\mathrm{CH}_{3}\right), 23$ $\left(\mathrm{CH}_{2}\right), 26\left(\mathrm{CH}_{2}\right), 30\left(\mathrm{CH}_{2}\right), 46\left(\mathrm{NCH}_{2}\right), 54\left(\mathrm{CH}_{2} \mathrm{~N}\right), 59$ $\left(\mathrm{CH}_{2} \mathrm{~N}\right.$ morph.), $66\left(\mathrm{OCH}_{2}\right.$ morph.), $67\left(\mathrm{OCH}_{2}\right.$ morph.). Found, \%: C 68.75, H 12.97; N 8.92. $\mathrm{C}_{9} \mathrm{H}_{19} \mathrm{NO}$. Calculated, \%: C 68.81, H 12.09; N 8.91.Yield 5 g (62\%), bp. $60^{\circ} \mathrm{C}(2 \mathrm{~mm} \cdot \mathrm{Hg}), \mathrm{n}_{D}^{20} 1.4111, \mathrm{~d}_{4}^{20}$.

N-Pentylethanolamine $\left(\mathrm{III}_{\mathrm{d}}\right), \mathrm{IR}\left(\mathrm{v}, \mathrm{cm}^{-1}\right): 3475(\mathrm{OH})$, $3400(\mathrm{NH}), 2920\left(\mathrm{CH}_{3}\right), 2820\left(\mathrm{CH}_{2}\right), 1225(\mathrm{C}-\mathrm{N}) .{ }^{1} \mathrm{H}$ NMR $\left(300 \mathrm{MHz}, \mathrm{D}_{2} \mathrm{O}\right) \delta: 0.87 \mathrm{t}\left(3 \mathrm{H}, \mathrm{CH}_{3}\right), 1.25 \mathrm{~m}(4 \mathrm{H}$, $\left.\mathrm{CH}_{2}\right), 1.45 \mathrm{~m}\left(2 \mathrm{H}, \mathrm{CH}_{2}\right), 2.5$ и $2.65 \mathrm{t}\left(4 \mathrm{H}, \mathrm{CH}_{2} \mathrm{~N}\right), 3.5-$ 3.6 t. t $\left(3 \mathrm{H}, \mathrm{NH}, \mathrm{NCH}_{2}\right), 4.75 \mathrm{~s}\left(3 \mathrm{H}, \mathrm{CH}_{2} \mathrm{OH}\right) .{ }^{13} \mathrm{C} \mathrm{NMR}$ $\left(300 \mathrm{MHz}, \mathrm{D}_{2} \mathrm{O}\right) \delta: 13.8\left(\mathrm{CH}_{3}\right), 22.4\left(\mathrm{CH}_{2}\right), 29.0\left(\mathrm{CH}_{2}\right)$, $29.5\left(\mathrm{CH}_{2}\right), 49.1(\mathrm{NHC}), 50.7(\mathrm{CHN}), 60.0(\mathrm{C}-\mathrm{OH})$. Mass spectrum (ES), m/z (I, \%): $131[\mathrm{M}]+(37) 100$ (100), 74 (100), 56 (100). Found, \%: C 65.02, H 13.01; N 10.73. $\mathrm{C}_{7} \mathrm{H}_{17} \mathrm{NO}$. Calculated, \%: C 64.12, H 12.97; N 10.68. Yield $2.98 \mathrm{~g}(76 \%)$, bp. $83^{\circ} \mathrm{C}-85^{\circ} \mathrm{C}(2 \mathrm{~mm} \cdot \mathrm{Hg})$, $\mathrm{n}_{D}^{20} 1.4382, \mathrm{~d}_{4}^{20} 0.8712$.

N-Pentylbenzylamine (III $)$, IR $\left(v, \mathrm{~cm}^{-1}\right): 2910\left(\mathrm{CH}_{3}\right)$, $2850\left(\mathrm{CH}_{2}\right), 1220(\mathrm{CN}) .{ }^{1} \mathrm{H}$ NMR $\left(300 \mathrm{MHz}, \mathrm{D}_{2} \mathrm{O}\right) \delta$ : $0.95 \mathrm{~m}\left(3 \mathrm{H}, \mathrm{CH}_{3}\right), 1.35 \mathrm{q}\left(2 \mathrm{H}, \mathrm{CH}_{2}\right), 1.45-1.6 \mathrm{~m}(7 \mathrm{H}$, $\left.\mathrm{NH}, \mathrm{CH}_{2}\right), 2.63 \mathrm{~m}\left(2 \mathrm{H}, \mathrm{NCH}_{2}\right), 3.8 \mathrm{~s}\left(2 \mathrm{H}, \mathrm{CH}_{2} \mathrm{Ph}\right), 7.15-$ $7.4\left(4 \mathrm{H}, \mathrm{H}\right.$ ar). ${ }^{13} \mathrm{C}$ NMR $\left(300 \mathrm{MHz}, \mathrm{CDCl}_{3}\right) \delta: 54.25$ $\left(\mathrm{CH}_{2} \mathrm{Ph}\right), 126.82,128.12,128.37,128.89\left(\mathrm{C}_{\mathrm{Ar}}\right)$. Found, \%: C 81.51, H 10.61; N 7.99. $\mathrm{C}_{12} \mathrm{H}_{19} \mathrm{~N}$. Calculated, \%: C 81.37, H 10.73; N 7.90.Yield 2.97 g (85\%), b.p. 112 $114(6 \mathrm{~mm} \cdot \mathrm{Hg}), \mathrm{n}_{D}^{20} 1.5073, \mathrm{~d}_{4}^{20} 0.9088$.

$\mathrm{N}$-(3-methylbutyl)diethylamine $\left(\mathrm{III}_{\mathrm{f}}\right), \operatorname{IR}\left(v, \mathrm{~cm}^{-1}\right)$ : $2940\left(\mathrm{CH}_{3}\right), 2860\left(\mathrm{CH}_{2}\right), 1227(\mathrm{C}-\mathrm{N}) .{ }^{1} \mathrm{H}$ NMR $(300$ $\left.\mathrm{MHz}, \mathrm{D}_{2} \mathrm{O}\right) \delta: 1.07 \mathrm{~d}\left(6 \mathrm{H}, \mathrm{CH}_{3}\right), 1.15 \mathrm{~m}\left(6 \mathrm{H}, \mathrm{CH}_{3}\right), 1.47$ $\left(2 \mathrm{H}, \mathrm{CH}_{2}\right), 1.79 \mathrm{~s}(1 \mathrm{H}, \mathrm{CH}), 2.5 \mathrm{q}$ and $2.65 \mathrm{~m}(6 \mathrm{H}$, $\left.\mathrm{CH}_{2} \mathrm{~N}\right) \cdot{ }^{13} \mathrm{C}$ NMR $\left(300 \mathrm{MHz}, \mathrm{D}_{2} \mathrm{O}\right) \delta: 14\left(\mathrm{CH}_{3}\right), 17$ $\left(\mathrm{CH}_{3}\right), 23\left(\mathrm{CH}_{3}\right), 26\left(\mathrm{CH}_{3}\right), 27\left(\mathrm{CH}_{2}\right), 36(\mathrm{CH}), 46\left(\mathrm{CH}_{2}\right)$, $47\left(\mathrm{CH}_{2}\right), 51\left(\mathrm{NCH}_{2}\right)$. Found, \%: C 75.63, H 14.48; N 9.62. $\mathrm{C}_{9} \mathrm{H}_{21} \mathrm{~N}$. Calculated, \%: C 75.54, N 14.67; N 9.78.Yield 8.57 g $(60 \%)$, bp. $139^{\circ} \mathrm{C}, \mathrm{n}_{D}^{20} 1.4109, \mathrm{~d}_{4}^{20}-$ 0.7779 .

$\mathrm{N}-\left(3\right.$-methylbutyl)piperidine (III $\mathrm{g}_{\mathrm{g}}$, IR $\left(v, \mathrm{~cm}^{-1}\right): 2920$ $\left(\mathrm{CH}_{3}\right), 2860\left(\mathrm{CH}_{2}\right), 1230(\mathrm{C}-\mathrm{N}) .{ }^{1} \mathrm{H}$ NMR $(300 \mathrm{MHz}$,
$\left.\mathrm{D}_{2} \mathrm{O}\right) \delta: 1 \mathrm{~d}\left(6 \mathrm{H}, \mathrm{CH}_{3}\right), 1.45 \mathrm{~s}(1 \mathrm{H}, \mathrm{CH}), 1.5-1.8 \mathrm{~m}(6 \mathrm{H}$, $\mathrm{CH}_{2}$ pip. $\mathrm{CH}_{2}$ alif.), $2.34 \mathrm{~m}\left(2 \mathrm{H}, \mathrm{NCH}_{2}\right), 2.4 \mathrm{~m}\left(6 \mathrm{H}, \mathrm{CH}_{2}\right.$ pip.). ${ }^{13} \mathrm{C}$ NMR $\left(300 \mathrm{MHz}, \mathrm{D}_{2} \mathrm{O}\right) \delta: 11.8\left(\mathrm{CH}_{3}\right), 18.0$ $\left(\mathrm{CH}_{3}\right), 25.5\left(\mathrm{CH}_{2}\right), 24.5(\mathrm{CH}), 26\left(\mathrm{CH}_{2}\right), 27\left(\mathrm{CH}_{2}\right.$ pip. $)$, $35(\mathrm{CH}), 54.4\left(\mathrm{CH}_{2} \mathrm{~N}\right.$ pip.), $55.5\left(\mathrm{CH}_{2} \mathrm{~N}\right.$ pip. $), 57.7$ $\left(\mathrm{NCH}_{2}\right.$ alif.). Found, \%: C 77.73, H 13.45; N 9.10. $\mathrm{C}_{10} \mathrm{H}_{21} \mathrm{~N}$. Calculated, \%: C 77.42, N 13.55; N 9.03.Yield $8.13 \mathrm{~g}(81 \%), \mathrm{bp} 60^{\circ} \mathrm{C}(2 \mathrm{~mm} \cdot \mathrm{Hg}), \mathrm{n}_{D}^{20} 1.4378, \mathrm{~d}_{4}^{20}-$ 0.8392 .

$\mathrm{N}-\left(3-\right.$ methylbutyl)morpholine $\left(\mathrm{III}_{\mathrm{h}}\right), \quad \mathrm{IR} \quad\left(v, \mathrm{~cm}^{-1}\right)$ : $2900\left(\mathrm{CH}_{3}\right), 2850\left(\mathrm{CH}_{2}\right), 1230(\mathrm{C}-\mathrm{N}) .{ }^{1} \mathrm{H}$ NMR $(300$ $\left.\mathrm{MHz}, \mathrm{D}_{2} \mathrm{O}\right) \delta: 1.05 \mathrm{~d}\left(6 \mathrm{H}, \mathrm{CH}_{3}\right), 1.5$ q. $\left(2 \mathrm{H}, \mathrm{CH}_{2}\right), 1.8 \mathrm{~s}$ $(1 \mathrm{H}, \mathrm{CH}), 2.4 \mathrm{~m}, 2.5 \mathrm{~m}\left(6 \mathrm{H}, \mathrm{CH}_{2} \mathrm{~N}\right.$ morph.), $3.17 \mathrm{~m}(4 \mathrm{H}$, $\mathrm{OCH}_{2}$ morph.). ${ }^{13} \mathrm{C}$ NMR (300 MHz, $\left.\mathrm{D}_{2} \mathrm{O}\right) \delta: 12\left(\mathrm{CH}_{3}\right)$, $17.7\left(\mathrm{CH}_{3}\right), 23.3(\mathrm{CH}), 26.6\left(\mathrm{CH}_{2}\right), 35.5\left(\mathrm{NCH}_{2}\right), 53$ ( $\mathrm{NCH}_{2}$ mor-ph.), 54 ( $\mathrm{CH}_{2} \mathrm{~N}$ morph.), 57 ( $\mathrm{CH}_{2} \mathrm{O}$ morph.), $67\left(\mathrm{CH}_{2} \mathrm{O}\right.$ morph.). Found, \%: C 69.01, H 12.34; N9.02. $\mathrm{C}_{9} \mathrm{H}_{19} \mathrm{NO}$. Calculated: $\mathrm{C} 68.81, \mathrm{H} 12.09$; $\mathrm{N}$ 8.91.Yield $4.42 \mathrm{~g}(71 \%)$, bp. $70^{\circ} \mathrm{C}(10 \mathrm{~mm} \cdot \mathrm{Hg}), \mathrm{n}_{D}^{20} 1.4381, \mathrm{~d}_{4}^{20}-$ 0.8935 .

$\mathrm{N}$-(3-methylbutyl)ethanolamine $\left(\mathrm{III}_{\mathrm{i}}\right), \operatorname{IR}\left(v, \mathrm{~cm}^{-1}\right)$ : $3440(\mathrm{OH}), 2910\left(\mathrm{CH}_{3}\right), 2860\left(\mathrm{CH}_{2}\right), 1225(\mathrm{CN}) .{ }^{1} \mathrm{H}$ NMR $\left(300 \mathrm{MHz}, \mathrm{D}_{2} \mathrm{O}\right) \delta: 0.9 \mathrm{~d}\left(6 \mathrm{H}, \mathrm{CH}_{3}\right), 1.3 \mathrm{~m}(2 \mathrm{H}$, $\left.\mathrm{CH}_{2}\right), 1.5 \operatorname{sep}(1 \mathrm{H}, \mathrm{CH}), 2.4-2.65 \mathrm{~m}\left(3 \mathrm{H}, \mathrm{CH}_{2} \mathrm{NH}\right), 3.5 \mathrm{t}$ $\left(2 \mathrm{H}, \mathrm{NCH}_{2}\right), 4.7 \mathrm{~s}\left(3 \mathrm{H}, \mathrm{CH}_{2} \mathrm{OH}\right) .{ }^{13} \mathrm{C}$ NMR $(300 \mathrm{MHz}$, $\left.\mathrm{D}_{2} \mathrm{O}\right)$ \&: $22.55\left(\mathrm{CH}_{3}\right), 22.47\left(\mathrm{CH}_{3}\right), 26.4(\mathrm{CH}), 38.22$ $\left(\mathrm{CH}_{2}\right), 50.95\left(\mathrm{CH}_{2} \mathrm{~N}\right), 59.94\left(\mathrm{CH}_{2} \mathrm{OH}\right)$. Found, \%: C 64.09, H 4.13; N 11.65. $\mathrm{C}_{7} \mathrm{H}_{17} \mathrm{NO}$. Calculated, \%: C 64.12, H 12.97; N 12.68.Yield $3.84 \mathrm{~g}(74 \%)$, bp. $77^{\circ} \mathrm{C}$ $78^{\circ} \mathrm{C}(2 \mathrm{~mm} \cdot \mathrm{Hg}), \mathrm{n}_{D}^{20} 1.4371, \mathrm{~d}_{4}^{20} 0.8772$.

$\mathrm{N}$-(3-methylbutyl)benzylamine $\left(\mathrm{III}_{\mathrm{k}}\right), \quad$ IR $\left(v, \mathrm{~cm}^{-1}\right)$ : $2920\left(\mathrm{CH}_{3}\right), 2840\left(\mathrm{CH}_{2}\right), 1225(\mathrm{C}-\mathrm{N}) .{ }^{1} \mathrm{H}$ NMR $(300$ $\left.\mathrm{MHz}, \mathrm{CDCI}_{3}\right) \delta: 0.95 \mathrm{t}\left(6 \mathrm{H}, \mathrm{CH}_{3}\right), 1.4 \mathrm{kv}\left(2 \mathrm{H}, \mathrm{CH}_{2}\right), 1.7$ sep $(1 \mathrm{H}, \mathrm{CH}), 2.7$ tr. $\left(2 \mathrm{H}, \mathrm{N}-\mathrm{CH}_{2}\right), 3.38 \mathrm{~s}\left(2 \mathrm{H}, \mathrm{PhCH}_{2}\right)$, 7.2 - $7.5 \mathrm{~m}(5 \mathrm{H}, \mathrm{H} \mathrm{Ar}) .{ }^{13} \mathrm{C}$ NMR $\left(300 \mathrm{MHz}, \mathrm{D}_{2} \mathrm{O}\right) \delta$ : $17.7\left(\mathrm{CH}_{3}\right), 22.7\left(\mathrm{CH}_{3}\right), 22.6\left(\mathrm{CH}_{2}\right), 39.27(\mathrm{CH}), 47.7$ $\left(\mathrm{N}-\mathrm{CH}_{2}\right), 54.25\left(\mathrm{PhCH}_{2}\right), 126.82,128.12,128.37,128.89$ (C Ar). Found, \%: C 81.62, H 10.59; N 7.81. $\mathrm{C}_{12} \mathrm{H}_{19} \mathrm{~N}$. Calculated, \%: C 81.37, H 10.73; N 7.90.Yield $4.53 \mathrm{~g}$ $(82 \%)$, bp. $78^{\circ} \mathrm{C}-79^{\circ} \mathrm{C}(2 \mathrm{~mm} \cdot \mathrm{Hg}), \mathrm{n}_{D}^{20} 1.4891, \mathrm{~d}_{4}^{20}-$ 0.8972 .

3-(N, N-diethylamino)propan-2-ol (VII $)$, IR $\left(v, \mathrm{~cm}^{-1}\right)$ : $3342(\mathrm{OH}), 2970\left(\mathrm{CH}_{3}\right), 2875(\mathrm{C}-\mathrm{N}), 1067(\mathrm{C}-\mathrm{O}) .{ }^{1} \mathrm{H}$ NMR $\left(300 \mathrm{MHz}, \mathrm{CDCI}_{3}\right) \delta: 0.9 \mathrm{t}\left(3 \mathrm{H}, \mathrm{CH}_{3}\right), 1.0 \mathrm{~d}(6 \mathrm{H}$, $\left.2 \mathrm{CH}_{3}\right), 2.1 \mathrm{t}\left(2 \mathrm{H}, \mathrm{NCH}_{2}\right), 2.25-2.5 \mathrm{~m} .\left(4 \mathrm{H}, 2 \mathrm{CH}_{2} \mathrm{~N}\right)$, 3.6 sep. $(1 \mathrm{H}, \mathrm{CH}), 3.7 \mathrm{~s}$.(wide) $(1 \mathrm{H}, \mathrm{OH}) .{ }^{13} \mathrm{C}$ NMR $(300$ $\left.\mathrm{MHz}, \mathrm{CDCI}_{3}\right) \delta: 20.0\left(\mathrm{CH}_{3}\right), 20.3\left(\mathrm{CH}_{3}\right), 21.4\left(\mathrm{CH}_{3}\right)$, $24.2\left(\mathrm{CH}_{2}\right), 49.5\left(\mathrm{NCH}_{2}\right), 55.8(\mathrm{C}-\mathrm{OH})$. Found, \%: C 64.03, H 16.82; N 13.75. $\mathrm{C}_{7} \mathrm{H}_{17}$ NO. Calculated, \%: C 64.13 , H 17.00; N 14.01.Yield 11.5 g (80\%), bp. $64^{\circ} \mathrm{C}-$ $65^{\circ} \mathrm{C}(15 \mathrm{~mm} \cdot \mathrm{Hg}), \mathrm{n}_{D}^{20} 1.4210$

3-Piperidinopropan-2-ol $\left(\mathrm{VII}_{\mathrm{b}}\right), \quad \mathrm{IR}\left(v, \mathrm{~cm}^{-1}\right): 3352$ (OH), $2934\left(\mathrm{CH}_{3}\right), 2855(\mathrm{CN}), 1072(\mathrm{C}-\mathrm{O}) .{ }^{1} \mathrm{H}$ NMR $\left(300 \mathrm{MHz}, \mathrm{CDCI}_{3}\right) \delta: 1.0 \mathrm{~d}\left(3 \mathrm{H}, \mathrm{CH}_{3}\right), 1.55-1.65 \mathrm{~m}(6 \mathrm{H}$, 
$\mathrm{CH}_{2}$ pip.), $2.8-2.9 \mathrm{~m}\left(4 \mathrm{HCH}_{2} \mathrm{~N}\right), 3.0$ d.d $\left(2 \mathrm{H}, \mathrm{NCH}_{2}\right)$, 3.5 sep $(1 \mathrm{H},-\mathrm{CH}), 3.7$ s.(waid), $(1 \mathrm{H}, \mathrm{OH}) .{ }^{13} \mathrm{C} \mathrm{NMR}$ (300 MHz,) $\mathrm{CDCI}_{3} \delta: 24\left(\mathrm{CH}_{3}\right), 24.5$ (C pip.), $24.7(\mathrm{C}$ pip.), 26.6 (C, pip.), $54.1\left(\mathrm{NCH}_{2}\right), 56.8$ (C-OH). Found, \%: C 67.08, H 11.9; N 9.69. $\mathrm{C}_{8} \mathrm{H}_{17} \mathrm{NO}$. Calculated, \%: C 67.18 , H 11.88; N 9.78.Yield 7.13 g $(96 \%)$, bp. $75^{\circ} \mathrm{C}$ $-76^{\circ} \mathrm{C}(20 \mathrm{~mm} \cdot \mathrm{Hg}) \mathrm{n}_{D}^{20} 1.4775$.

3-Morpholinopropan-2-ol $\left(\mathrm{VII}_{\mathrm{c}}\right)$, IR $\left(v, \mathrm{~cm}^{-1}\right): 3441$ $(\mathrm{OH}), 2965\left(\mathrm{CH}_{3}\right), 2854(\mathrm{CN}), 1063(\mathrm{C}-\mathrm{O}) .{ }^{1} \mathrm{H}$ NMR (300 MHz,) $\mathrm{CDCI}_{3}, \delta: 1.0 \mathrm{~d}\left(3 \mathrm{H}, \mathrm{CH}_{3}\right), 2.17$ d.d $(2 \mathrm{H}$, $\left.\mathrm{NCH}_{2}\right), 2.4$ - $2.5 \mathrm{~m}\left(4 \mathrm{H}, \mathrm{CH}_{2} \mathrm{~N}\right.$ morph.), $3.57 \mathrm{~m}(4 \mathrm{H}$, $\left.\mathrm{CH}_{2} \mathrm{O}\right), 3.7$ sep. $(1 \mathrm{H}, \mathrm{CH}), 3.8$ s.(wiat) $(1 \mathrm{H}, \mathrm{OH}) .{ }^{13} \mathrm{C}$ NMR $\left(300 \mathrm{MHz}, \mathrm{CDCI}_{3}\right) \delta: 22.3\left(\mathrm{CH}_{3}\right), 53\left(\mathrm{CH}_{2} \mathrm{~N}\right.$ morph.), $56\left(\mathrm{NCH}_{2}\right), 56.6(\mathrm{C}-\mathrm{OH}), 66\left(\mathrm{OCH}_{2}\right.$ morph.). Found, \%: C 56.96, H 10.14; N 9.55. $\mathrm{C}_{7} \mathrm{H}_{15} \mathrm{O}_{2} \mathrm{~N}$. Calculated, \%: C 57.96, H 10.34; N 9.65.Yield 14.2 g (98\%), bp. $70^{\circ} \mathrm{C}-71^{\circ} \mathrm{C}(3 \mathrm{~mm} \cdot \mathrm{Hg}), \mathrm{n}_{D}^{20} 1.4590$.

3-Benzilaminopropan-2-ol $\left(\mathrm{VII}_{\mathrm{d}}\right), \operatorname{IR}\left(v, \mathrm{~cm}^{-1}\right): 3250$ $(\mathrm{OH}), 2980\left(\mathrm{CH}_{3}\right), 2830(\mathrm{CN}), 1058(\mathrm{C}-\mathrm{O}) .{ }^{1} \mathrm{H}$ NMR $\left(300 \mathrm{MHz}, \mathrm{CDCI}_{3}\right) \delta: 1.17 \mathrm{~d}\left(3 \mathrm{H}, \mathrm{CH}_{3}\right), 2.5$ - 2.6 t.d $(2 \mathrm{H}$, $\left.\mathrm{NCH}_{2}\right), 3.32-3.36$ s.(wiat) $(2 \mathrm{H}, \mathrm{NHOH}), 3.78 \mathrm{~d}(2 \mathrm{H}$, $\left.\mathrm{PhCH}_{2}\right), 3.86$ sep. $(1 \mathrm{H}, \mathrm{CH}), 7.28$ - $7.29 \mathrm{~m}\left(5 \mathrm{H}, n-\mathrm{C}_{6} \mathrm{H}_{5}\right)$. ${ }^{13} \mathrm{CNMR}\left(300 \mathrm{MHz}, \mathrm{CDCI}_{3}\right) \delta: 22.2\left(\mathrm{C}^{1}\right), 53.3 \mathrm{~m}\left(\mathrm{C}^{3}\right)$, $56.6 \mathrm{~m}\left(\mathrm{C}^{2}\right), 65.5\left(\mathrm{RhCH}_{2}\right), 126,127,140$ (CAr). Found, \%: C 72.67, H 9.15; N 8.54. $\mathrm{C}_{10} \mathrm{H}_{15} \mathrm{ON}$. Calculated, \%: C 72.75, H 9.08; N 8.48.Yield 9.9 g $(60 \%)$, bp. $126^{\circ} \mathrm{C}$ $-127^{\circ} \mathrm{C}(2 \mathrm{~mm} \cdot \mathrm{Hg}), \mathrm{n}_{D}^{20} 1.5270$.

Bis-1,3-(N,N-diethylamino)propan-2-ol ( $\left.\mathrm{VIII}_{\mathrm{a}}\right), \mathrm{IR}(v$, $\left.\mathrm{cm}^{-1}\right)$ : $3415(\mathrm{OH}), 2940\left(\mathrm{CH}_{3}\right), 2805(\mathrm{CN}), 1115(\mathrm{C}-\mathrm{O})$. ${ }^{1} \mathrm{H}$ NMR $\left(300 \mathrm{MHz}, \mathrm{D}_{2} \mathrm{O}\right) \delta: 0.95 \mathrm{~m}\left(12 \mathrm{H}, 4 \mathrm{CH}_{3}\right), 2.34-$ $2.52 \mathrm{~m}\left(12 \mathrm{H}, 6 \mathrm{NCH}_{2}\right), 3.28 \mathrm{~s}(1 \mathrm{H},-\mathrm{OH}), 3.5 \mathrm{sep} .(1 \mathrm{H}$, $\mathrm{OCH}) .{ }^{13} \mathrm{C}$ NMR $\left(300 \mathrm{MHz}, \mathrm{D}_{2} \mathrm{O}\right) \delta: 10\left(\mathrm{CH}_{3}\right), 47.1 \mathrm{~m}$ (C3), $57.05 \mathrm{~m}\left(\mathrm{NCH}_{2}\right), 66.49$ (C2). Found, \%: C 65.42, $\mathrm{H}$ 12.97; N 13.93. $\mathrm{C}_{11} \mathrm{H}_{26} \mathrm{~N}_{2} \mathrm{O}$. Calculated, \%: C 65.37, $\mathrm{H}$ 12.86; N 13.85.Yield $9.1 \mathrm{~g}(93 \%)$, bp. $122^{\circ} \mathrm{C}(10 \mathrm{~mm} \cdot \mathrm{Hg}$.) $\mathrm{n}_{D}^{20} 1.4571, \mathrm{~d}_{4}^{20} 0.9101$.

Bis-1,3-piperidinopropan-2-ol $\left(\mathrm{VIII}_{\mathrm{b}}\right)$, IR $\left(v, \mathrm{~cm}^{-1}\right)$ : $3410(\mathrm{OH}), 2980\left(\mathrm{CH}_{3}\right), 2800(\mathrm{CN}), 1125(\mathrm{C}-\mathrm{O}) .{ }^{1} \mathrm{H}$ NMR (300 MHz, $\left.\mathrm{CDCl}_{3}, \delta\right): 1.28-1.53 \mathrm{~m}\left(12 \mathrm{H}, \mathrm{CH}_{2} \mathrm{~N}\right.$ pip. $\mathrm{NCH}_{2}$ aliph.), $2.18 \mathrm{~s}(\mathrm{OH}), 3.75$ sept $(\mathrm{OCH}) .{ }^{13} \mathrm{C}$ NMR (300 MHz, D $2 \mathrm{O}) \delta: 24.5\left(\mathrm{CH}_{2}\right.$ pip.), $26.6\left(\mathrm{CH}_{2}\right.$ pip.), $54.4\left(\mathrm{CH}_{2} \mathrm{~N}\right.$ pip. $), 63.33\left(\mathrm{~N}-\mathrm{CH}_{2}\right.$ alf.), $64.44(\mathrm{OCH})$. Found, \%: C 66.13, H 11.62; N 12.05. $\mathrm{C}_{13} \mathrm{H}_{26} \mathrm{~N}_{2} \mathrm{O}$. Calculated, \%: C 69.03, H 11.49; N 12.38.Yield 12 g (90\%), bp. $135^{\circ} \mathrm{C}-136^{\circ} \mathrm{C}(3 \mathrm{~mm} \cdot \mathrm{Hg}.) \mathrm{n}_{D}^{20} 1.4955, \mathrm{~d}_{4}^{20} 1.1137$.

Bis-1,3-morpholinopropan-2-ol $\left(\mathrm{VIII}_{\mathrm{c}}\right), \operatorname{IR}\left(v, \mathrm{~cm}^{-1}\right)$ : $3422(\mathrm{OH}), 2940\left(\mathrm{CH}_{3}\right), 2805(\mathrm{CN}), 1110(\mathrm{C}-\mathrm{O}) .{ }^{1} \mathrm{H}$ NMR (300 MHz, $\left.\mathrm{D}_{2} \mathrm{O}\right) \delta$ : $2.17 \mathrm{~d}(1 \mathrm{H}, \mathrm{OH}), 2.2-2.5 \mathrm{~m}$ $\left(8 \mathrm{H}, \mathrm{CH}_{2}, \mathrm{NCH}_{2} \mathrm{cycl}\right), 3.5 \mathrm{t}\left(4 \mathrm{H}, \mathrm{OCH}_{2} \mathrm{cycl}\right), 3.7 \mathrm{~s}(\mathrm{OCH})$. ${ }^{13} \mathrm{C}$ NMR $\left(300 \mathrm{MHz}, \mathrm{D}_{2} \mathrm{O}\right) \delta: 53\left(\mathrm{NCH}_{2}\right), 62\left(\mathrm{CH}_{2} \mathrm{~N}\right.$ cycl.), $63(\mathrm{OCH}), 66\left(\mathrm{OCH}_{2}\right)$. Found, \%: C 57.30, $\mathrm{H}$ 9.38; $\mathrm{N}$ 12.29. $\mathrm{C}_{11} \mathrm{H}_{22} \mathrm{~N}_{2} \mathrm{O}_{3}$. Calculated, \%: C 57.42, $\mathrm{H}$ 9.56; N 12.17.Yield 11.6 g (87\%), bp. $142^{\circ} \mathrm{C}-144^{\circ} \mathrm{C}(2$ $\mathrm{mm} \cdot \mathrm{Hg}$.) $\mathrm{n}_{D}^{20} 1.4358, \mathrm{~d}_{4}^{20} 0.9818$.
Bis-1,3-(N,N-diethylamino)propane (IX $), \operatorname{IR}\left(v, \mathrm{~cm}^{-1}\right)$ : $2980\left(\mathrm{CH}_{3}\right), 1275,865(\mathrm{CN}) .{ }^{1} \mathrm{H}$ NMR $\left(300 \mathrm{MHz}, \mathrm{D}_{2} \mathrm{O}\right)$ $\delta$ : $1.1 \mathrm{t}\left(12 \mathrm{H}, \mathrm{CH}_{3}\right), 1.6$ q. $\left(2 \mathrm{H}, \mathrm{CH}_{2}\right), 2.46-2.62$ sq. $(12 \mathrm{H}$, $\left.\mathrm{CH}_{2} \mathrm{NCH}_{2}\right) .{ }^{13} \mathrm{C} \mathrm{NMR}\left(300 \mathrm{MHz}, \mathrm{CDCl}_{3}\right) \delta: 11.4\left(\mathrm{CH}_{3}\right)$, $25\left(\mathrm{CH}_{2}\right), 47\left(\mathrm{CH}_{2} \mathrm{~N}\right), 52\left(\mathrm{NCH}_{2}\right)$. Found, \%: C 70.73, 70.79; $\mathrm{H} 14.26,14.20 ; \mathrm{N} \mathrm{15.21,} \mathrm{15.19.} \mathrm{C}_{11} \mathrm{H}_{26} \mathrm{~N}_{2}$. Calculated, \%: C 70.97, H 13.96; N 15.05.Yield $11.7 \mathrm{~g}$ (63\%), bp. $85^{\circ} \mathrm{C}(20 \mathrm{~mm} \cdot \mathrm{Hg}), \mathrm{n}_{D}^{20} 1.4295, \mathrm{~d}_{4}^{20} 0.8392$.

Bis-1,3-piperidinopropan $\left(\operatorname{IX}_{\mathrm{b}}\right), \operatorname{IR}\left(v, \mathrm{~cm}^{-1}\right)$ : 1278 , $850(\mathrm{C}-\mathrm{N}) .{ }^{1} \mathrm{H}$ NMR $\left(300 \mathrm{MHz}, \mathrm{D}_{2} \mathrm{O}\right) \delta: 1.43-1.54 \mathrm{~m}$ $\left(14 \mathrm{H}, \mathrm{CH}_{2}\right), 2.33-2.43 \mathrm{~m}\left(12 \mathrm{H}, \mathrm{CH}_{2} \mathrm{NCH}_{2}\right) .{ }^{13} \mathrm{C} \mathrm{NMR}$ (300 MHz, $\left.\mathrm{D}_{2} \mathrm{O}\right) \delta: 22.0,23.0,26.0\left(\mathrm{CH}_{2}\right.$, pip. $\mathrm{CH}_{2}$ alif.), $54\left(\mathrm{CH}_{2} \mathrm{~N}\right.$ pip.), 57 ( $\mathrm{NCH}_{2}$ aliph.). Found, \%: $\mathrm{C} 74.49$, 74.51; H 12.43, 12.49; N 13.15, 13.21. $\mathrm{C}_{13} \mathrm{H}_{26} \mathrm{~N}_{2}$. Calculated, \%: C 74.29, H 13.37; N 13.33.Yield 8.13 g (66\%), bp. $116^{\circ} \mathrm{C}(2 \mathrm{~mm} \cdot \mathrm{Hg}) . \mathrm{n}_{D}^{20} 1.4773, \mathrm{~d}_{4}^{20} 0.9354$,

Bis-1,3-morpholinopropane (IX $\left.\mathrm{I}_{\mathrm{c}}\right), \mathrm{IR}\left(v, \mathrm{~cm}^{-1}\right)$ : 1235, $850(\mathrm{C}-\mathrm{N}) .{ }^{1} \mathrm{H}$ NMR $\left(300 \mathrm{MHz}, \mathrm{D}_{2} \mathrm{O}\right) \delta: 1.43-1.54$ and $1.56-1.7 \mathrm{~m}\left(14 \mathrm{H}, \mathrm{CH}_{2}\right), 2.33-2.43 \mathrm{~m}\left(12 \mathrm{H}, \mathrm{CH}_{2}\right.$ morph., $\mathrm{NCH}_{2}$ alif.). ${ }^{13} \mathrm{C} \mathrm{NMR}\left(300 \mathrm{MHz}, \mathrm{D}_{2} \mathrm{O}\right) \delta: 24.0$ $\left(\mathrm{CH}_{2}\right.$ alif.), 54.0, $56.0\left(\mathrm{CH}_{2} ; \mathrm{NCH}_{2}\right.$ morph.), $66\left(\mathrm{CH}_{2} \mathrm{O}\right.$ morph.). Found,\%: C 61.65, 61.58, H 10.13, 10.20; N 13.15, 13.13. $\mathrm{C}_{11} \mathrm{H}_{22} \mathrm{~N}_{2} \mathrm{O}_{2}$. Calculated, \%: C 61.69, $\mathrm{H}$ $10.27 ; \mathrm{N} 13.08$.Yield $8.12 \mathrm{~g}(60 \%)$, bp. $125^{\circ} \mathrm{C}(1$ $\mathrm{mm} \cdot \mathrm{Hg}), \mathrm{n}_{D}^{20} 1.4781, \mathrm{~d}_{4}^{20} 1.0367$.

\section{Results and Discussion}

We have directed our efforts on the region-selective synthesis of amino- and bis-amino alcohols, which may have antibacterial and anticorrosion properties. Interactions of a number of initial amines $\left(\mathrm{I}_{\mathrm{a}-\mathrm{e}}\right)$ with brom-pentanes $\left(\mathrm{II}_{\mathrm{a}-\mathrm{b}}\right)$ result in $\mathrm{N}$-alkylamines ( $\mathrm{III}_{\mathrm{a}-\mathrm{k}}$ ) (Scheme 1).

The synthesis of target products $\left(\mathrm{III}_{\mathrm{a}-\mathrm{m}}\right)$ was carried out inaqueous medium at $50^{\circ} \mathrm{C}-90^{\circ} \mathrm{C}$ for $6-9 \mathrm{~h}$. The yields of target pro-ducts were in the range $60 \%$ - 98\%. Synthesis of alkyl amines $\left(\mathrm{III}_{\mathrm{a}}, \mathrm{III}_{\mathrm{b}}, \mathrm{III}_{\mathrm{f}}, \mathrm{III}_{\mathrm{g}}, \mathrm{IX}_{\mathrm{a}}, \mathrm{IX}_{\mathrm{b}}\right)$ was performed at $50^{\circ} \mathrm{C}$ for $8 \mathrm{~h}$ at a molar ratio of initial components (amines: pentylbromide or dibromopropane) equal to $5: 1$. The reaction of monoetha-nolamine $\left(\mathrm{I}_{\mathrm{d}}\right)$ and morpholine $\left(\mathrm{I}_{\mathrm{c}}\right)$ with pentylbromides $\left(\mathrm{II}_{\mathrm{a}, \mathrm{b}}\right)$ goes at a relatively high temperature of $60^{\circ} \mathrm{C}$ and long duration $(9 \mathrm{~h})$ at optimum component ratio of $5: 1$. The reaction between benzylamine $\left(\mathrm{I}_{\mathrm{e}}\right)$ and pentylbromides $\left(\mathrm{II}_{\mathrm{a}, \mathrm{b}}\right)$ is better to carry out at the even higher temperature $\left(90^{\circ} \mathrm{C}\right)$ for $9 \mathrm{~h}$ and the same ratio of reactants. Another possible ap-

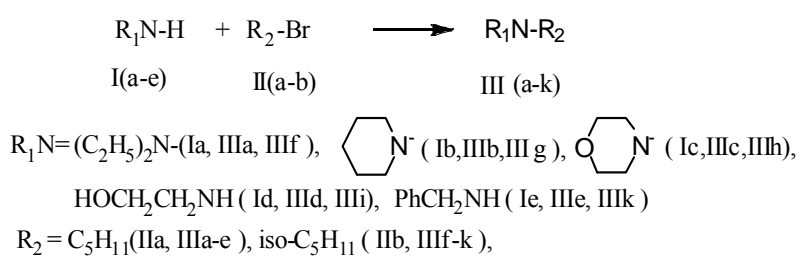

Scheme 1. Synthesis of substituted N-alkylaminesby the alkylation with brompentanes. 
proach has been realized by us in obtaining aminoalcohols VII $_{(\mathrm{a}-\mathrm{d})}$, bis-aminopropane-2-ols $\left(\mathrm{VIII}_{\mathrm{a}-\mathrm{c}}\right)$ and bisaminopropanes $\left(\mathrm{IX}_{\mathrm{a}-\mathrm{c}}\right)$. We carried out regioselective oxirane ring opening of propylene oxide (IV), epichlorohydrin (V) and 1.3-dibromopropan (VI) as a result of interaction with various amines in aqueous medium according to Scheme 2.

The interaction of propylene oxide (IV) and epichlorohydrin $(\mathrm{V})$ with diethylamine $\left(\mathrm{I}_{\mathrm{a}}\right)$ was carried out with stirring the mixture of reagents in the water by heating at $50^{\circ} \mathrm{C}$ for 9 hours. The optimum ratio of initial components in this case was as follows: diethylamine/propylene oxide $=1.1: 1$; diethylamine/epichlorohydrin $=5: 1$. The reaction of diethylamine with propylene oxide formed secondary amino alcohol $\left(\mathrm{VII}_{\mathrm{a}}\right)$, while the interacttion with epichloro-hydrin leads to the formation of bisamino alcohol $\left(\right.$ VIII $\left._{a}\right)$, with the yields $80 \%$ and $98 \%$, respectively. The reactions of piperidine $\left(\mathrm{I}_{\mathrm{b}}\right)$, morpholine $\left(\mathrm{I}_{\mathrm{c}}\right)$ and benzylamine $\left(\mathrm{I}_{\mathrm{e}}\right)$ with epoxy compounds were carried out at the higher temperature $\left(90^{\circ} \mathrm{C}\right)$ for $9 \mathrm{~h}$. The optimal relations between the components of amines $\left(\mathrm{I}_{\mathrm{b}}\right.$, $\mathrm{I}_{\mathrm{c}}$ and $\mathrm{I}_{\mathrm{e}}$ ) and propylene oxide (IV) were 1.1-1, and in the case of epichlo-rohydrin 5:1. Yields of the products with in the limits of $60 \%-90 \%$ were achieved. Earlier aminoalcohols $\left(\mathrm{VII}_{\mathrm{a}-\mathrm{d}}\right.$ and $\left.\mathrm{VIII}_{\mathrm{a}-\mathrm{c}}\right)$ were obtained in organic solvents in the presence of catalysts such as salts, metal triflates as complexing agents and others with the yields within $75 \%$ - 85\% [15-24].

Aminolysis of epoxides could precede regionselectively under (a) or against (b) Krasusky rule [25]. The value of $\delta$ protons $\mathrm{CH}\left(\mathrm{C}^{2}\right)$ group in ${ }^{1} \mathrm{H}$ NMR spectrum of compound $\left(\mathrm{VII}_{\mathrm{a}}\right)$ is in the range of $3.60 \mathrm{ppm}$, indicating a shift of the signals in a weak field. This result shows that the $\mathrm{OH}$ group is located at the carbon atom $\mathrm{C}^{2}$. Pro-ton signals of the methyl group at the atom $\mathrm{C}^{1}$ are also shifted toward weaker fields under the influence of the electronegative oxygen atom in the possession of the neighboring atom $\left(\mathrm{C}^{2}\right)(1.35 \mathrm{ppm})$. Similarly, proton signals of $\mathrm{CH}$ groups of compounds $\left(\mathrm{VII}_{\mathrm{b}-\mathrm{d}}\right)$ and $\mathrm{CH}_{2}$ groups of compounds $\left(\mathrm{VIII}_{\mathrm{a}-\mathrm{c}}\right.$ ) are shifted downfield in comparison with the reference materials. Good correlation with published data for compounds of similar structure $[14,15,17,19]$ also shows in favor of the structure.

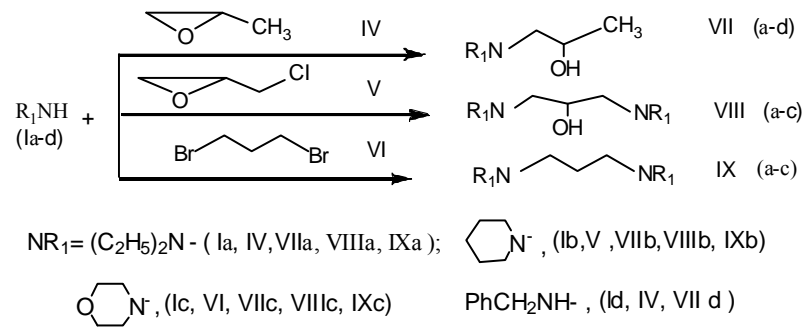

Scheme 2. Regioselectiveoxirane ring opening with amines and synthesis aminoalkohols and diamines.
Chromatographic analysis showed the presence of only one regioisomer for obtained compounds. Thus, the openings of the epoxy fragments in compounds (IV, V) with amines $\left(\mathrm{I}_{\mathrm{a}-\mathrm{e}}\right)$ is in accordance with Krasusky rule and follow the path(a) with the formation of secondary alcohols derivatives region selectively.

Original amines $\left(\mathrm{I}_{\mathrm{a}-\mathrm{d}}\right)$ are good or partially soluble in water, with the possible formation of the corresponding unstable hydroxides $\left[\mathrm{RR}_{1} \mathrm{NH}_{2}\right]^{+} \mathrm{OH}^{-}$. It is possible and the formation of quaternary ammonium salts $\left[\mathrm{RR}_{1} \mathrm{NH}_{2}\right]^{+} \mathrm{Br}^{-}$ as a result of interaction between amines $\left(\mathrm{I}_{\mathrm{a}-\mathrm{d}}\right)$ and brompentanes $\left(\mathrm{II}_{\mathrm{a}, \mathrm{b}}\right)$ or 1.3 dibromopropane $\left(\mathrm{II}_{\mathrm{c}}\right)$. Obtained hydroxides and quaternary ammonium salts are readily soluble in water and not on-lyplay the role of phase transfer catalysts in the reaction of the starting amines $\left(\mathrm{I}_{\mathrm{a}-\mathrm{e}}\right)$ with brompentanes $\left(\mathrm{II}_{\mathrm{a}, \mathrm{b}}\right)$ and also could perform catalysis for opening oxiranesin aqueous media. Epichlorohydrin and 1.3 dibromo-propane also form quaternary am-monium salts next type $\left\{\left[\mathrm{R}_{2} \mathrm{NHR}\right]^{+}-\mathrm{Cl}^{-}\right\}$, that are phase transfer catalysts. The opening of epoxide ring for propylene oxide and epichlorohydrin under the action of these PTC-catalysts proceeds on well-known scheme [5] for the $\mathrm{S}_{\mathrm{N}} 2$ mechanism with the formation of amino and bisaminopropane derivatives.

The obtained hydroxyamino- and bis-amino-compounds are promising for use as an anti-bacterial and antirust agent for oil production.

\section{Conclusion}

An operationally simple and environmentally benign protocol for the ring opening of epoxides with aliphatic amines has been developed. The reactions proceeded smoothly under mild conditions in water to afford $a$ amino alcohols in high yields with excellent regioselectivities. Also substituted amines were also obtained in environmentally acceptable conditions in the water by the al-kylation with brompentanes.

\section{REFERENCES}

[1] M. A. Fernandez-Rodriguezs, Q. L. Shen and J. F. Hartwig, "A General and Long-Livedcatalysttor the Palladium-Catalyzedcoupling of Arylhalideswith Thiols," Journal of the American Chemical Society, Vol. 128, No. 7, 2006, pp. 2180-2181. doi:10.1021/ja0580340

[2] L. Chao-Jun, "Organic Reactions in Agueousmrdia with a Focus on Carbon-Carbon Bond Formations: A Decade Update," Chemical Reviews, Vol. 105, No. 8, 2005, pp. 3095-3165. doi:10.1021/cr030009u

[3] R. N. Butler and A. G. Coyne, "Water: Natural's Reaction Enforcer-Comparative Effects for Organic Synthesis 'inWater' and 'on-Water'," Chemical Reviews, Vol. 110, No 10, 2010, pp. 6302-6337. doi:10.1021/cr100162c

[4] Z.-L. Shen, K. KuaKiatGoh, H.-L. Cheong, C. H. A. Wong, Y.-C. Lai, Y.-S. Yang and T.-P. Loh, "Synthesis 
of Water-Tolerant Indium Homo-Enolate in Agueous Media and Its Application in the Synthesis of 1,4-Dicarbonyl Compounds via Palladium-Catalyzed Coupling with Acidc Hloride," Journal of the American Chemical Society, Vol. 132, No. 45, 2010, pp. 15852-15855. doi:10.1021/ja106925f

[5] N. Azizi and M. R. Saidi, "Highly Chemo-Selective Addition of Amines to Epoxides in Water," Organic Letters, Vol. 7, No. 17, 2005, pp. 3649-3651.

[6] G. Evans, T. J. K. Gibbs, R. L. Zenkins, S. J. Coles, M. B. Hursthouse, A. J. Platts and N. C. O. Tomkinson, "Kinetics of Iminium Ion Catalysis," Angewandte Chemie International Edition, Vol. 47, No. 15, 2008, pp. 2820-2823.

[7] D. Zhu, L. Xu, F. Wu and B. Wan, "A Mild and Efficient Copper-Catalyzed Coupling of Aryl Iodides and Thiols Using an Oximephosphine Oxide Ligand," Tetrahedron Letters, Vol. 47, No. 32, 2006, pp. 5781-5784.

[8] L. Rout, T. K. Sen and T. Punniyamurthy, "Efficient CuO-Nanoparticlecatalyzed C-S Cross-Copling of Thiols with Iodobenzene," Angewandte Chemie International Edition, Vol. 46, No. 29, 2007, pp. 5583-5586.

[9] K. Hu, K. E. Krakowiask, J. S. Bradshaw, N. K. Dalley, G. Xue and R. M. Izzat, "Synthesys of Chiral Azamacrocycles Using the Bis( $\alpha$-chloroacetamide)s Derived from Chiral 1,2-Di-phenylethy-lenediamine," Journal of Heterocyclic Chemistry, Vol. 36, No. 2, 1999, pp. 347354.

[10] Y. Nagasaki, E. Honzawa, M. Kato and T. Tsuruta, "Novel Synthesis of a Mac-Romonomer Having Organosilyl and Amino Groups," Journal of Macromolecular Science: Pure and Applied Chemistry, Vol. 29, No. 6, 1992, pp. 457-470.

[11] M. K. Denk, K. Hatono and A. J. Lough, "Synthesis and Characterization of a Carbine- $\mathrm{SO}_{2}$," European Journal of Inorganic Chemistry, Vol. 2003, No. 2, 2003, pp. 224231. doi:10.1002/ejic.200390030

[12] J. F. Lehman, S. G. Urguhart, L. E. Ennis, A. P. Hitchcock, K. Hatano, S. Gupta and M. K. Denk, "Core Excitation Spectroscopy of Stable Cyclic Diamino-Carbenes, -Silylenes, and -Germylenes," Organometalliks, Vol. 18, No. 10, 1999, pp. 1862-1872.

[13] J. Huskens and M. T. Reetz, "Observation of stereotopic group recognition in chiral borate complexes in solution," European Journal of Organic Chemistry, Vol. 1999, No. 8, 1999, pp. 1775-1786. doi:10.1002/(SICI)1099-0690(199908)1999:8<1775::AID -EJOC1775>3.0.CO;2-1

[14] N. G. Di Masi, F. P. Intini, C. Pacifico, L. Maresca and G. S. Natile, "Synthesis and Spectroscopic and Structural Characterization of Different Configuretions of Tridentate
Amine Ligand N,N',N" Trimet-Hyldiethylentriamine Coordinated to Platinum," Inorganica Chimica Acta, Vol. 310, No. 52, 2000, pp. 27-33.

[15] M. Chini, P. Crotti and F. Macchia, "Metal Salts as New Catalysts for Mild and Efficient Aminolysis of Oxiranes," Tetrahedron Letters, Vol. 31, No. 32, 1990, pp. 46614664.

[16] E. Cherbulies, A. Yazgi and J. Rabinowitz, "Recherchessur la Formation et la Transformation des Esters XXIV. Note sur la Préparation de l'Acide Diéthylami-no-1-propyl-2-phosphorique," Helvetica Chimica Acta, Vol. 44, No. 4, 1961, pp. 1164-1166.

[17] A. T. Placzek, J. L. Donelson, R. Trivedi, R. A. Gibbs, and S. K. De, "Scandium Triflate as an Efficient and Useful Catalyst for the Synthesis of $\beta$-Amino Alcohols by Regioselective," Tetrahedron Letters, Vol. 46, No. 52 , 2005, pp. 9029-9034.

[18] C. M. Kleiner and P. R. Schreiner, "Hydrophobic Amplification of Noncovalent Organocatalysis," Chemical Communications, No. 41, 2006, pp. 4315-4317. doi: $10.1039 / \mathrm{b} 605850 \mathrm{~g}$

[19] R. G. Griot and A. J. Frey, "The Formation of Cyclols from N-Hydroxyacyllactams," Tetrahedron, Vol. 19, No. 11, 1963, pp. 1661-1673.

[20] N. B. Chapman and D. J. Triggle, "Di-N-Substituted 2Halogenoethylamines. Part VI. NN-dialkyl-(or N-alkyl)2-alkyl(or aryl or arylalkyl) Derivatives: Synthesis, Reactivity, and Pharmacology," Journal of the Chemical Society, 1963, pp. 1385-1400. doi:10.1039/jr9630001385

[21] A. A. Potechin and A. O. Saphronov, "Ring-Chain tautomerism of Substituted Hydrazones. N-Benzyl-(N-oxyal$k y l$ hydrazones of Aromatic Carbonyl Compounds," The Journal of Organic Chemistry (Russian), Vol. 17, 1981, pp. 379-387.

[22] V. R. Geartner, "Substituent Entropy-Controlled Strained Ring-Chain Equilibria," Journal of Organic Chemistry, Vol. 33, No. 2, 1968, pp. 523-530. doi:10.1021/jo01266a010

[23] H. K. Kochetkov and L. A. Vorotnikova, "Several Derivatives of 1,2,3-Triami-Nopropane," Journal of Computational Chemistry (Russian), Vol. 29, No. 3, 1959, pp. 532-535.

[24] A. N. Smirnov, E. G. Pomerahceva and I. F. Spasskaya, "Synthesis of O-Alkyl-N,N-al-kylideneisoureas," Journal of Organic Chemistry (Russian), Vol. 34, No. 11, 1964, pp. 3370-3387.

[25] D. N. Kirk, "Selectivity in Reaction of Epoxides," Chemistry and Industry, Vol. 3, 1973, pp. 109-116. 